CLINICAL STUDY

\title{
Observing pretibial myxedema in patients with Graves' disease using digital infrared thermal imaging and high-resolution ultrasonography: for better records, early detection, and further investigation
}

\author{
Shyang-Rong Shih ${ }^{1,2,3}$, Mao-Shin Lin ${ }^{2,3}$, Hung-Yuan Li ${ }^{2,3}$, Hsin-Yi Yang ${ }^{4}$, Yung-Lien Hsiao ${ }^{2}$, Met-Ting Chang ${ }^{2}$, \\ Chung-Ming Chen ${ }^{1}$ and Tien-Chun Chang ${ }^{2,3,4}$ \\ ${ }^{1}$ Institute of Biomedical Engineering, National Taiwan University, No.1, Sec.1, Jen-Ai Road, Taipei 100, Taiwan ROC, ${ }^{2}$ Department of Internal Medicine, \\ National Taiwan University Hospital, 7 Chung-Shan South Road, Taipei 100, Taiwan ROC, ${ }^{3}$ National Taiwan University College of Medicine, Taipei 100, \\ Taiwan ROC and ${ }^{4}$ Center of Anti-Aging and Health Consultation, National Taiwan University Hospital, Taipei 10O, Taiwan ROC \\ (Correspondence should be addressed to T-C Chang at Department of Internal Medicine, National Taiwan University Hospital; \\ Email: tienchunchang@ntu.edu.tw)
}

\begin{abstract}
Objective: Pretibial myxedema (PM) is a manifestation of Graves' disease (GD). Currently, its diagnosis depends on physicians' observation and biopsy. No satisfactory, objective, and non-invasive tool is available to record and investigate lesions. Digital infrared thermal imaging (DITI) detects surface temperature, and sonography reflects composition changes in soft tissue. This study was aimed to observe changes in DITI and sonography in PM, and to evaluate their clinical usefulness.

Methods: Nineteen GD patients with PM, 22 GD patients with mild diffuse non-pitting edema over lower legs, 46 GD patients with normal appearance of lower legs, and 14 normal volunteers were recruited for observation with DITI; $8,21,21$, and 11 of them respectively also received soft tissue sonography for investigating the pathogenesis of DITI change.

Results: Lower leg temperatures of normal volunteers decreased gradually from proximal to distal parts. In all 19 patients with PM, DITI showed abnormally low focal temperatures over the lesions. In GD patients with mild diffuse non-pitting edema and GD patients with normal appearance of lower legs, DITI showed abnormally low focal temperature in 90.9 and $65.2 \%$ of the patients respectively. Areas of clinically visible PM and low focal temperature detected by DITI were sonographically characterized with increased skin thickness, hypoechoic substance deposition in the cutaneous tissue, and blurred boundary lines between dermis and subcutaneous tissue. TSH receptor antibody level correlated positively and significantly with skin thickness change and adjusted temperature difference between the center of temperature defect and the surrounding skin $(P=0.046$ and 0.033 respectively).

Conclusions: By using DITI and sonography, we detected characteristic changes in PM. These techniques are helpful in recording and may be useful tools to detect early changes of PM.
\end{abstract}

European Journal of Endocrinology 164 605-611

\section{Introduction}

Graves' disease (GD) was first described by Parry in 1825 (1). It is characterized by thyrotoxicosis and goiter. Patients may have ophthalmopathy and dermopathy. The pathogenesis of GD involves an autoimmune process: T lymphocytes are sensitized, and B lymphocytes are stimulated to synthesize antibodies against the TSH receptor on follicular cells, which increases the growth and function of follicular cells and thus leads to hyperthyroidism (2). The prevalence of GD is $2.7 \%$ in women and about one-tenth as frequent in men (3).

Infiltrative dermopathy occurs in about 0.5 to $4.3 \%$ of patients with a history of thyrotoxicosis and 13\% of those with severe Graves' ophthalmopathy (4). It is characterized by non-pitting edema, most commonly appearing in the pretibial area (2). The pathogenesis of Graves' dermopathy also involves TSH receptor antibodies, which recognize the TSH receptor in the skin tissue (2). The downstream lymphokine activation of fibroblasts results in the overproduction and deposition of hyaluronic acid and chondroitin sulfate in the dermis and subcutaneous tissue, causing compression of dermal lymphatics and non-pitting edema (2). Nodules and plaques may form in chronic lesions (2). Dermopathy was classified into four forms according to its appearance: non-pitting edema accompanied by typical skin color change (diffuse form), plaque form, 
nodular form, and elephantiasis (5). Treatment and follow-up often require months to years $(6,7)$. Some studies obtained leg circumference, skin examination results, and photographs for outcome evaluation (8). However, measuring the leg circumference was difficult for recording plaque and nodular lesions; photographs could be interfered with light and were unable to record changes under the skin surface. Another study obtained the physicians' documentation in charts and patients' questionnaires as the outcomes (9). However, this method of evaluation was subjective. Therefore, we needed a more objective method to record and analyze lesions of pretibial myxedema (PM).
Digital infrared thermal imaging (DITI) is a modern technique used to record the temperature of the body surface $(10,11)$. It has been applied to evaluate Graves' ophthalmopathy, dry eye, diabetic neuropathy, and breast cancer (12-16). For example, thermal imaging of feet helped to detect thermoregulatory sweating abnormality in diabetic feet (15). Remote sensor thermometry proved better for the diagnosis of dry eye disease (14). In breast tumors, DITI identified $96.7 \%$ of malignancies and was thought to be a valuable adjunct to mammography and ultrasonography (17). In Graves' dermopathy, deposition of glycosaminoglycan (GAG) may result in temperature decrease. Thus, DITI of the

Table 1 Basic characteristics, temperatures, and sonographic characteristics of patients with Graves' dermopathy.

\begin{tabular}{|c|c|c|c|c|c|}
\hline & $\begin{array}{l}\text { Typical Graves' } \\
\text { dermopathy }\end{array}$ & $\begin{array}{l}\text { GD patients with mild } \\
\text { diffuse non-pitting } \\
\text { edema over lower legs }\end{array}$ & $\begin{array}{l}\text { GD patients with } \\
\text { normal appearance } \\
\text { of lower legs }\end{array}$ & $\begin{array}{l}\text { Healthy } \\
\text { volun- } \\
\text { teers }\end{array}$ & $P$ value \\
\hline Number of subjects & 19 & 22 & 46 & 12 & \\
\hline Age (years) & $42 \pm 11$ & $45 \pm 13$ & $42 \pm 11$ & $35 \pm 9$ & 0.078 \\
\hline \multicolumn{6}{|l|}{ Sex } \\
\hline Men & $4(21.1 \%)$ & $3(13.6 \%)$ & $6(13.0 \%)$ & $4(28.6 \%)$ & 0.498 \\
\hline Women & $15(78.9 \%)$ & $19(86.4 \%)$ & $40(87.0 \%)$ & $10(71.4 \%)$ & \\
\hline Duration of GD (months) & $59.9 \pm 59.6$ & $47.6 \pm 71.2$ & $53.2 \pm 52.2$ & & 0.958 \\
\hline Graves' ophthalmopathy & $88.2 \%$ & $73.7 \%$ & $50 \%$ & & 0.02 \\
\hline Smoking & $5.6 \%$ & $23 \%$ & $13.6 \%$ & 0 & 0.379 \\
\hline $\mathrm{fT}_{4}(\mathrm{ng} / \mathrm{dl})$ & $1.64 \pm 1.29$ & $2.15 \pm 1.24$ & $2.4 \pm 1.68$ & & 0.202 \\
\hline $\mathrm{TSH}(\mathrm{mU} / \mathrm{l})$ & $1.33 \pm 2.85$ & $0.43 \pm 1.05$ & $0.34 \pm 1.03$ & & 0.084 \\
\hline AMA (IU/ml) & $1064.5 \pm 759.7$ & $478.5 \pm 710.6$ & $699.5 \pm 721.2$ & & 0.215 \\
\hline TBII (\%) & $69.8 \pm 27.1$ & $56.2 \pm 28.8$ & $54.4 \pm 26.1$ & & 0.151 \\
\hline \multicolumn{6}{|l|}{ Drugs } \\
\hline$\beta$-blockers & $50 \%$ & $50 \%$ & $63.6 \%$ & & 0.461 \\
\hline Oral glucocorticoid & $5.9 \%$ & $21.4 \%$ & $2.9 \%$ & & 0.085 \\
\hline Topical glucocorticoid & $29.4 \%$ & 0 & 0 & & 0.001 \\
\hline Anti-histamine & $17.7 \%$ & $7.1 \%$ & $20.6 \%$ & & 0.614 \\
\hline Ear temperature $\left({ }^{\circ} \mathrm{C}\right)$ & $37.2 \pm 0.2$ & $36.9 \pm 0.3$ & $36.8 \pm 0.3$ & $36.8 \pm 0.4$ & 0.021 \\
\hline $\begin{array}{l}\text { Temperature defect noted in either } \\
\text { one of the legs }\end{array}$ & $100.0 \%$ & $90.9 \%$ & $65.2 \%$ & 0 & $<0.001^{\mathrm{a}}$ \\
\hline $\begin{array}{l}\text { Temperature difference between the } \\
\text { center of temperature defect and } \\
\text { normal skin }\left({ }^{\circ} \mathrm{C}\right)^{\mathrm{b}}\end{array}$ & $0.80 \pm 0.48$ & $0.57 \pm 0.32$ & $0.25 \pm 0.27$ & & $<0.001$ \\
\hline $\begin{array}{l}\text { Average temperatures of the } \\
\text { temperature defect on DITI }\left({ }^{\circ} \mathrm{C}\right)^{\mathrm{C}}\end{array}$ & $28.61 \pm 1.35$ & $29.02 \pm 1.07$ & $28.84 \pm 1.05$ & & 0.607 \\
\hline \multicolumn{6}{|c|}{ Sonography measure skin thickness at point $A$} \\
\hline $\begin{array}{l}\text { Average thickness of dermis and } \\
\text { epidermis }\end{array}$ & $0.19 \pm 0.02$ & $0.18 \pm 0.03$ & $0.19 \pm 0.02$ & $0.17 \pm 0.02$ & 0.111 \\
\hline $\begin{array}{l}\text { Average thickness of subcutaneous, } \\
\text { dermis, and epidermis }\end{array}$ & $0.77 \pm 0.22$ & $0.56 \pm 0.15$ & $0.56 \pm 0.15$ & $0.59 \pm 0.13$ & 0.015 \\
\hline \multicolumn{6}{|c|}{ Sonography measure skin thickness at point $\mathrm{C}$} \\
\hline $\begin{array}{l}\text { Average thickness of dermis and } \\
\text { epidermis }\end{array}$ & $0.26 \pm 0.10$ & $0.18 \pm 0.03$ & $0.19 \pm 0.05$ & $0.16 \pm 0.01$ & $<0.001$ \\
\hline $\begin{array}{l}\text { Average thickness of subcutaneous, } \\
\text { dermis, and epidermis }\end{array}$ & $1.02 \pm 0.38$ & $0.75 \pm 0.22$ & $0.65 \pm 0.17$ & $0.70 \pm 0.10$ & 0.002 \\
\hline $\begin{array}{l}\text { Blurred boundary lines between } \\
\text { dermis and subcutaneous tissue }\end{array}$ & $100.0 \%$ & $59.1 \%$ & $81.8 \%$ & $36.4 \%$ & 0.008 \\
\hline $\begin{array}{l}\text { Decreased echogenicity of lower part } \\
\text { of the shin skin compared with upper } \\
\text { part of the shin skin }\end{array}$ & $100.0 \%$ & $100.0 \%$ & $90.9 \%$ & $18.2 \%$ & $<0.001$ \\
\hline
\end{tabular}

$\mathrm{fT}_{4}$, free thyroxine; AMA, anti-thyroid microsomal antibody; TBII, TSH-binding inhibitory immunoglobulin; Point $\mathrm{A}$, upper one-fourth between the fibular head and lateral malleolus; Point $\mathrm{C}$, lower one-fourth between the fibular head and lateral malleolus.

a Include healthy volunteers or not, both were $<0.001$.

${ }^{\mathrm{b}}$ Temperature difference between lesions and normal skin $=$ (temperature at point $4+$ temperature at point 7$) / 2-$ temperature at point 1.

${ }^{\mathrm{c}}$ Average temperatures of the temperature defect on $\mathrm{DITI}\left({ }^{\circ} \mathrm{C}\right)=($ temperature at point $3+$ temperature at point $2+$ temperature at point $1+$ temperature at point $5+$ temperature at point 6$) / 5$. 
skin of Graves' dermopathy was considered to be different from DITI of the normal skin. The aim of this study was to investigate whether DITI and highresolution sonography were potentially helpful in recording Graves' dermopathy.

\section{Materials and methods}

This study was approved by the Institutional Review Board of the National Taiwan University Hospital (protocol number: 200811040R) and was registered on Clinicaltrials.gov (protocol number: NCT01182584).

\section{Patients}

We recruited 87 GD patients presented to the outpatient clinics of National Taiwan University Hospital and 14 normal volunteers from 2007 to 2010. Of the $87 \mathrm{GD}$ patients, 7 had diffuse form of PM, 2 had nodular form, and 10 had plaque form of Graves' dermopathy. In other 68 GD patients, 22 had mild diffuse non-pitting edema over lower legs without typical skin color change, which made the diagnosis of dermopathy equivocal, and 46 had normal appearance of lower legs. Detail characteristics were listed in Table 1. As to other diseases which might affect leg condition, one patient with nodular type of PM had diabetes under medical control. Among patients with mild diffuse non-pitting edema, two had pre-diabetes and one had Sjögren's syndrome. Among GD patients with normal appearance of lower leg, one had pre-diabetes, one had Sjögren's syndrome, and one had hypertension. None of them were diagnosed with peripheral arterial occlusive disease or arteritis. Some patients with Graves' dermopathy received systemic corticosteroid therapy after DITI had been performed $(5,18)$. Eight patients with PM, 21 patients with mild diffuse non-pitting edema, 21 patients with normal appearance of lower legs, and 11 normal volunteers also received soft tissue sonography for investigating DITI change. Graves' orbitopathy was diagnosed if patients had exophthalmos, diplopia, or any item included in the clinical activity score introduced by Mourits et al. (19) and revised by an ad hoc international committee, i.e. presence of pain on eye movement, spontaneous retrobulbar pain, conjunctiva injection, eyelid erythema, chemosis, and swelling of the caruncle or eyelid (20).

\section{Methods}

Patients or healthy volunteers sat quietly for more than 5 min in a temperature-controlled dark room maintained at around $23{ }^{\circ} \mathrm{C}$. Next, DITI was obtained using the DITI System, Spectrum 9000 MB Series (United Integrated Service, Taipei Hsien, Taiwan). The system was calibrated before each examination. After the thermometer sensor was placed on the focus of the measurement target, the infrared energy that radiated from the measurement target was sensed and converted into an electrical signal. The signal was connected to a computer that automatically plotted the thermal density. In colored pictures, grayish white, red, brown, yellow, green, blue, purple, and black represented temperatures from high to low. In black-and-white pictures, bright to dark colors represented temperatures from high to low. Local temperatures of 14 points above and around the lesions were measured in patients with Graves' dermopathy (Fig. 1). Soft tissue sonography was performed using the LOGIQ 5 Pro echo machine and the 12L probe (GE Medical System, Taipei, Taiwan). The skin of the anterior aspect of the lower legs between the tibia and fibula was observed. The skin thickness of cutaneous and subcutaneous tissue was recorded at three points: upper one-fourth (point A), mid (point B), and lower one-fourth (point C) between the fibular head and lateral malleolus.

This study followed the ethical standards of the Declaration of Helsinki. The study participants provided written and informed consent after the doctors explained the nature and possible consequences of the study.

\section{Analysis}

We observed the differences on DITI and soft tissue sonography in different groups of participants. All variables were summarized as mean and s.D. One-way ANOVA and $t$-test were applied to compare various factors between groups in Table 1. Multivariate logistic regression was performed to analyze the relationship between TSH-binding inhibitory immunoglobulin

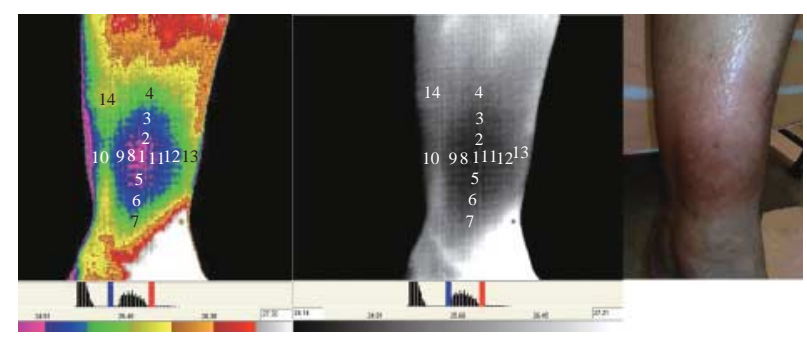

Figure 1 Local temperatures of 14 points above and around the lesions of the lower legs were measured with the Digital Infrared Thermal Image System. Left: color picture: grayish white, red, brown, yellow, green, blue, purple, and black represented temperatures from high to low. Middle: black-and-white picture, bright to dark colors represented temperatures from high to low. Point 1 represented the temperature in the center of the lesion. Points 3, 6, 9, and 12 represented temperatures near the edge of and over the lesion. Points 4, 7, 10, and 13 represented temperatures near the edge of and outside the lesion. Point 2 represented the temperature in the middle of points 1 and 3 ; point 8 , that in the middle of points 1 and 9 ; point 5 , that in the middle of points 1 and 6; and point 11, that in the middle of points 1 and 12 . Point 14 represented the temperature of normal skin at the left upper side of the lesion. Right: photograph of the corresponding lesion of the leg. 
(TBII), temperature difference between the center of temperature defect and the surrounding skin, thickness of epidermis, dermis and subcutaneous tissue, and body temperatures. Adjusted temperature difference between the center of temperature defect and normal skin was then generated according to the regression coefficient. Linear regression models were applied to analyze the relationship between various factors. Age and sex were regarded as potential confounders and were adjusted. A $P$ value $<0.05$ was considered statistically significant. The statistical analyses were performed with Stata/SE 9.0 for Windows (StataCorp LP, College Station, TX, USA).

\section{Results}

\section{DITI of the pretibial area of normal volunteers}

In normal volunteers, DITI showed a smooth temperature gradient that gradually decreased from the proximal to the distal portion of the limbs. The best focus of this DITI system was obtained at $25 \mathrm{~cm}$ distance. If we tried to include the whole length of the lower legs, the distance between the legs and the lens was around $50 \mathrm{~cm}$, thermal images became blurred, and the temperatures at lateral aspects of the legs interfered with the background. If we focused on the lower part of the lower leg, the distance between the legs and the lens was around $25 \mathrm{~cm}$, and the thermal images were clear.

\section{DITI of pretibial myxedema}

DITI of patients with plaque and nodular forms of dermopathy revealed that the temperatures over the lesion were lower than those of the surrounding normal skin (Fig. 2). In patients with diffuse form of dermopathy, areas of low focal temperature were noted on the DITI. Temperatures at the longitudinal midline of the lesions could be easily recorded. However, recording temperatures at the transverse line of the lesion was difficult and might be less precise because in some cases, the whole transverse aspect of the lesion could not be included in one photograph, and the recorded temperatures near the edge of the objects on the thermal image could be interfered with the background. Therefore, we chose points over the longitudinal midline of the lesions, including points $4,3,2,1,5,6$, and 7 , on Fig. 1, to record the average temperatures of the lesions and the temperature differences between the lesion and normal skin. The average temperature of the lesion was defined as ((summation of temperatures at points 3,2 , 1,5 , and 6)/5). The average temperature of normal skin near the lesion site was defined as ((temperature at point $4+$ temperature at point 7 )/2). The temperature difference between the lesion and normal skin was defined as ((temperature at point $4+$ temperature at point 7)/2)-temperature at point 1 . Results were shown in Table 1.

\section{DITI of the pretibial area in GD patients with mild diffuse non-pitting edema over lower legs}

Temperature defects at either one of the legs could be detected in 90.9\% (20/22) patients in this group (Table 1 and Fig. 3).

\section{DITI of the pretibial area in GD patients with normal appearance of lower legs}

Temperature defects at either one of the legs could be detected in $65.2 \%(30 / 46)$ patients in this group (Table 1 and Fig. 3).

\section{Observation of the correlation of DITI changes with soft tissue sonography}

In patients with diffuse form of PM, DITI showed an uneven temperature distribution. Soft tissue sonography showed that the skin thickness increased noticeably from points $\mathrm{A}$ to $\mathrm{B}$ to $\mathrm{C}$, the boundary lines between dermis and subcutaneous tissue became blurred, and the amount of substances with low echogenicity deposited in the cutaneous tissue (dermis and epidermis) increased downward the legs (Fig. 3). In patients with plaque form dermopathy, DITI showed low focal temperature over the plaques. Sonography showed

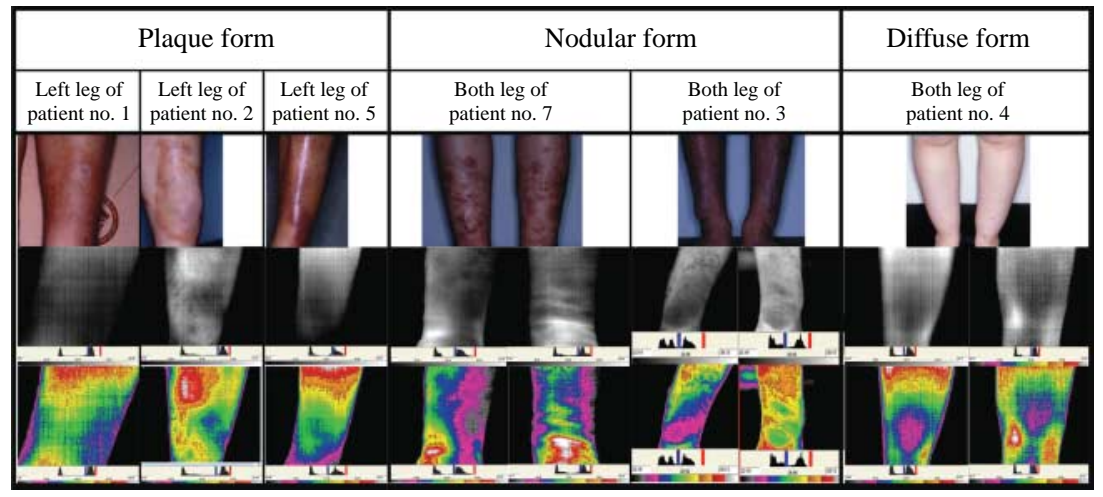

Figure 2 Digital infrared thermal image of pretibial myxedema. 


\begin{tabular}{|c|c|c|c|c|c|}
\hline & \multirow[t]{2}{*}{ Normal } & \multicolumn{2}{|c|}{$\begin{array}{l}\text { Graves' disease } \\
\text { with pretibial myxedema }\end{array}$} & \multicolumn{2}{|c|}{$\begin{array}{l}\text { Graves' disease } \\
\text { without pretibial myxedema }\end{array}$} \\
\hline & & Diffuse form & Plaque form & Abnormal DITI & Normal DITI \\
\hline Photo & & & & & \\
\hline DITI & & & & & \\
\hline $\begin{array}{c}\text { Echo } \\
\text { (point A) }\end{array}$ & & & & & \\
\hline Skin thick & $0.77 \mathrm{~cm}$ & $0.52 \mathrm{~cm}$ & $0.99 \mathrm{~cm}$ & $0.63 \mathrm{~cm}$ & $0.48 \mathrm{~cm}$ \\
\hline $\begin{array}{c}\text { Echo } \\
\text { (point C } \\
\text { or area of } \\
\text { abnormal } \\
\text { DITI) }\end{array}$ & & & & & 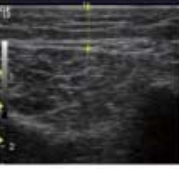 \\
\hline Skin thick & $0.82 \mathrm{~cm}$ & $0.83 \mathrm{~cm}$ & $1.15 \mathrm{~cm}$ & $0.75 \mathrm{~cm}$ & $0.60 \mathrm{~cm}$ \\
\hline
\end{tabular}

Figure 3 Correlation of digital infrared thermal image and sonography of pretibial area. Point A means upper one-fourth between the fibular head and lateral malleolus. Point $\mathrm{C}$ means lower one-fourth between the fibular head and lateral malleolus. increased skin thickness, blurred boundary lines between the dermis and subcutaneous tissue, and deposition of substances with low echogenicity in the cutaneous tissue over the plaques (Fig. 3). In patients with mild diffuse pretibial non-pitting edema and normal skin color (i.e. equivocal diagnosis of dermopathy), echogenicity was lower in the cutaneous tissue with or without increased skin thickness and blurred boundary lines between the dermis and subcutaneous tissue, over where areas of low focal temperature were shown on DITI (Fig. 3). In patients with GD with normal appearance of the lower legs, the echogenicity was lower in the cutaneous tissue, and the boundary lines between dermis and subcutaneous tissue were blurred with or without increased skin thickness, over where areas of low focal temperature were shown on DITI (Fig. 3); the echographic change here was to a lesser degree than that of clinically apparent dermopathy. In normal subjects, DITI showed a smooth temperature gradient that gradually decreased from the proximal to the distal portion of the limbs, as mentioned above. The echogenicity of the cutaneous tissue decreased, and the boundary lines between the dermis and subcutaneous tissue increased only a small percentage of healthy subjects (both $P<0.05$ compared with GD patients with normal appearance of the lower legs; Table 1). The ratio of lower to upper shin skin thickness in healthy subjects was less than that in patients with GD with normal appearance of the lower legs with no statistic significance $(P=0.151$ if epidermis and dermis were measured, and $P=0.611$ if skin thickness was measured from epidermis to subcutaneous tissue), which probably was related to the small number of healthy volunteers.

Multivariate logistic regression performed to analyze the relationship between TBII, temperature difference between the center of temperature defects and the surrounding skin, thickness of epidermis to subcutaneous tissue, and body temperature, using TBII as a dependent variable, results in regression coefficients of 16.2, 29.9, and 8.8 respectively. According to this ratio, we generated an adjusted temperature difference between the center of temperature defect and the surrounding skin $=$ (temperature difference between the center of temperature defect and the surrounding skin $\left.\left({ }^{\circ} \mathrm{C}\right)\right)+1.8 \times($ thickness of epidermis to subcutaneous tissue $(\mathrm{cm}))+0.5 \times\left(\right.$ body temperature $\left({ }^{\circ} \mathrm{C}\right)$ ). This adjusted temperature difference correlated positively and significantly with TBII (correlation coefficient: $0.127, P=0.033$ ). Besides, statistic analysis showed that the ratio of (thickness of dermis and epidermis at point $\mathrm{C}$ )/(thickness of dermis and epidermis at point A) was positively and significantly correlated with TBII and adjusted temperature difference $(P=0.046$ and 0.004 respectively) .

\section{Discussion}

Patients with Graves' dermopathy had higher levels of TBII and higher frequency of Graves' ophthalmopathy than patients in other groups in our study (Table 1). 
This observation was compatible with the literature: high levels of TSH receptor antibodies and Graves' ophthalmopathy were almost always present in patients with thyroid dermopathy $(2,6)$. Our study showed that the results of DITI and soft tissue sonography of PM were different from those of normal persons. These techniques were objective tools for recording and detecting early changes of PM. The temperatures of the lesions were lower than those of normal skin, which was supposed to be due to GAG deposition. Currently, the hypothetic pathogenesis of Graves' dermopathy involves TSH receptor antibodies, the downstream lymphokine activation of fibroblasts, and the overproduction and deposition of hyaluronic acid and chondroitin sulfate in the dermis and subcutaneous tissue (2). This GAG deposition might lead to poor heat transmission from arteries deep in the legs. This effect was supposed to overwhelm the heat produced from inflammation, and to result in focal low temperature on the surface.

Areas of low focal temperature were detected over plaques and nodules. This observation was compatible with the findings of soft tissue sonography. The low echogenicity of substances in the cutaneous tissue may represent GAG deposition and contribute to the increased cutaneous thickness (Fig. 3). This finding was compatible with the study by Salvi et al. (21) in 1994. They found increased skin thickness in $76 \%$ of patients with clinically suspected Graves' dermopathy. Another sonographic finding, i.e. the blurred boundary lines between the dermis and subcutaneous tissue, might be explained by host's response to inflammation or GAG deposition. In patients with diffuse form of PM, areas of low focal temperature were also detected on DITI, whereas soft tissue sonography showed the same typical findings as in plaques and nodules. This observation could also be explained by GAG deposition, i.e. the temperature difference between areas of low focal temperature caused by GAG deposition and other skin areas was not as large as that observed in patients with plaque forms, the skin did not protrude as much, and the color of the skin did not differ as much. This hypothesis could also explain defects on DITI in GD patients with normal appearance of the lower legs and in patients with equivocal diagnosis of dermopathy. In these patients, DITI revealed areas of low focal temperature over the pretibial skin, where soft tissue sonography showed typical changes comparable with those of patients with visible dermopathy, but to a lesser degree. It was suggested that there might be more patients with GD who have pretibial GAG accumulation than we know. If GAG accumulation was severe enough to be visible, patients could have been diagnosed with Graves' dermopathy in clinical practice. This finding was supported by higher levels of TSH receptor antibodies in patients with Graves' dermopathy than in patients without dermopathy. In other words, the prevalence of dermopathy may be higher than
0.5-4.3\% among patients with GD. In a study of forearm skin biopsy of nine patients with GD without PM, dense mucin deposition in the papillary dermis was noted in five patients (22). The authors suggested that deposition of mucopolysaccharide was a widespread phenomenon in GD, and that clinical PM represented changes at a later stage of the disease, which correlated with our study and hypothesis. However, further studies are necessary to support this hypothesis.

The pitfall of this study was mainly the lack of tissue proof in patients with GD. Because some studies suggested that lesions might develop in surgical scars and trauma might exacerbate Graves' dermopathy, we could not decide to perform biopsy in our study $(23,24)$. Magnetic resonance imaging (MRI) could also be considered for its typical presentation of Graves' dermopathy (25). Only two patients underwent MRI in our study initially, but we found that this method failed to detect the lesion due to small changes and difficulties tin localizing the lesion noted at DITI. Therefore, we performed soft tissue sonography.

This study showed that the findings of DITI and soft tissue sonography of the pretibial area were different in normal persons and patients with Graves' dermopathy. These methods are good objective tools for recording Graves' dermopathy. DITI findings of the pretibial area were also different in normal persons and some Graves' dermopathy patients without visible dermopathy, which suggested 'subclinical' dermopathy. It would be worthwhile to conduct further large-scale studies to verify our observation.

\section{Declaration of interest}

The authors declare that there is no conflict of interest that could be perceived as prejudicing the impartiality of the research reported.

\section{Funding}

This research did not receive any specific grant from any funding agency in the public, commercial or not-for-profit sector.

\section{Author contribution statement}

S-R Shih involved in the study design, statistic analysis, and paper writing. M-S Lin performed the study design. H-Y Li involved in the statistic analysis. H-Y Yang was the study executor, and performed image analysis. Y-L Hsiao was the study executor, and involved in paper writing. M-T Chang was the study executor, and performed image analysis. C-M Chen involved in the supervision and paper revision. T-C Chang performed the study design, supervision, and paper revision.

\section{References}

1 Parry CH. Disease of the heart. In Collections from the Unpublished Writings, vol 2, pp 111-125. London: Underwoods, 1825.

2 Greenspan FS. The thyroid gland. In Basic and Clinical Endocrinology, pp 215-294. Eds FS Greenspan \& DG Gardner. New York, NY: McGraw-Hill Companies, 2004. 
3 Tunbridge WM, Evered DC, Hall R, Appleton D, Brewis M, Clark F, Evans JG, Young E, Bird T \& Smith PA. The spectrum of thyroid disease in a community: the Whickham survey. Clinical Endocrinology $1977 \quad 7$ 481-493. (doi:10.1111/j.1365-2265. 1977.tb01340.x)

4 Fatourechi V. Pretibial myxedema: pathophysiology and treatment options. American Journal of Clinical Dermatology 20056 295-309. (doi:10.2165/00128071-200506050-00003)

5 Fatourechi V, Pajouhi M \& Fransway AF. Dermopathy of Graves' disease (pretibial myxedema). Review of 150 cases. Medicine 1994 73 1-7. (doi:10.1097/00005792-199401000-00001)

6 Vahab F. Pretibial myxedema: pathophysiology and treatment options. American Journal of Clinical Dermatology 20056 295-309. (doi:10.2165/00128071-200506050-00003)

7 Chang TC, Koa SC \& Huang KM. Octreotide and Graves' ophthalmopathy and pretibial myxoedema. BMJ 1992304158. (doi:10.1136/bmj.304.6820.158)

8 Rotman-Pikielny P, Brucker-Davis F, Turner ML, Sarlis NJ \& Skarulis MC. Lack of effect of long-term octreotide therapy in severe thyroid-associated dermopathy. Thyroid 200313 465-470. (doi:10.1089/105072503322021124)

9 Schwartz KM, Fatourechi V, Ahmed DD \& Pond GR. Dermopathy of Graves' disease (pretibial myxedema): long-term outcome. Journal of Clinical Endocrinology and Metabolism $2002 \mathbf{8 7}$ 438-446. (doi:10.1210/jc.87.2.438)

10 Jiang LJ, Ng EY, Yeo AC, Wu S, Pan F, Yau WY, Chen JH \& Yang Y. A perspective on medical infrared imaging. Journal of Medical Engineering and Technology 200529 257-267. (doi:10.1080/ 03091900512331333158)

11 Jones BF. A reappraisal of the use of infrared thermal image analysis in medicine. IEEE Transactions on Medical Imaging 1998 17 1019-1027. (doi:10.1109/42.746635)

12 Chang TC, Hsiao YL \& Liao SL. Application of digital infrared thermal imaging in determining inflammatory state and follow-up effect of methylprednisolone pulse therapy in patients with Graves' ophthalmopathy. Graefe's Archive for Clinical and Experimental Ophthalmology $2008 \mathbf{2 4 6}$ 45-49. (doi:10.1007/s00417-0070643-0)

13 Shih SR, Li HY, Hsiao YL \& Chang TC. The application of temperature measurement of the eyes by digital infrared thermal imaging as a prognostic factor of methylprednisolone pulse therapy for Graves' ophthalmopathy. Acta Ophthalmologica 2010 88 e154-e159. (doi:10.1111/j.1755-3768.2010.01941.x)

14 Singh G \& Bhinder HS. Comparison of noncontact infrared and remote sensor thermometry in normal and dry eye patients. European Journal of Ophthalmology 200515 668-673.
15 Sun PC, Lin HD, Jao SH, Ku YC, Chan RC \& Cheng CK. Relationship of skin temperature to sympathetic dysfunction in diabetic at-risk feet. Diabetes Research and Clinical Practice 200673 41-46. (doi:10.1016/j.diabres.2005.12.012)

16 Agnese DM. Advances in breast imaging. Surgical Technology International $20051451-56$.

17 Arora N, Martins D, Ruggerio D, Tousimis E, Swistel AJ, Osborne MP \& Simmons RM. Effectiveness of a noninvasive digital infrared thermal imaging system in the detection of breast cancer. American Journal of Surgery 2008196 523-526. (doi:10.1016/j. amjsurg.2008.06.015)

18 Chang TC, Huang KM, Hsiao YL, Tseng SS \& Kao SC. Relationships of orbital computed tomographic findings and activity scores to the prognosis of corticosteroid therapy in patients with Graves' ophthalmopathy. Acta Ophthalmologica Scandinavica $1997 \mathbf{7 5}$ 301-304. (doi:10.1111/j.1600-0420.1997.tb00779.x)

19 Mourits MP, Koornneef L, Wiersinga WM, Prummel MF, Berghout A \& van der Gaag R:. Clinical criteria for the assessment of disease activity in Graves' ophthalmopathy: a novel approach. British Journal of Ophthalmology 198973 639-644. (doi:10.1136/ bjo.73.8.639)

20 Wartofsky L. Classification of eye changes of Graves' disease. Thyroid 19922 235-236. (doi:10.1089/thy.1992.2.235)

21 Salvi M, DeChiara F, Gardini E, Minelli R, Bianconi L, Alinovi A, Ricci R, Neri F, Tosi C \& Roti E. Echographic diagnosis of pretibial myxedema in patients with autoimmune thyroid disease. European Journal of Endocrinology 1994131 113-119. (doi:10.1530/eje.O. 1310113)

22 Wortsman J, Dietrich J, Traycoff RB \& Stone S. Preradial myxedema in thyroid disease. Archives of Dermatology 1981117 635-638. (doi:10.1001/archderm.117.10.635)

23 Wright AL, Buxton PK \& Menzies D. Pretibial myxedema localized to scar tissue. International Journal of Dermatology 19902954. (doi:10.1111/j.1365-4362.1990.tb03758.x)

24 Singh SP, Ellyin F, Singh SK \& Yoon B. Elephantiasis-like appearance of upper and lower extremities in Graves' dermopathy. American Journal of the Medical Sciences 1985290 73-76. (doi:10. 1097/00000441-198508000-00006)

25 Davies TF \& Larsen PR. Thyrotoxicosis. In Williams Textbook of Endocrinology, pp 374-422. Eds RH William \& PR Larsen. Philadelphia, PA: Elsevier Science, 2003.

Received 23 January 2011

Accepted 25 January 2011 